\title{
MÁS ALLÁ DEL MERCADO ALTERNATIVO DE VALORES: EN LA BÚSQUEDA DE UNA MAYOR GESTIÓN PROFESIONAL EN LAS EMPRESAS PERUANAS
}

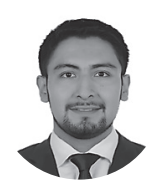

\author{
EFRAÍN M. CONTRERAS
}

- Profesor de Fundamentos de las Finanzas

Carrera de Administración y Finanzas

Facultad de Negocios, UPC

Actualmente, se han generado diferentes opiniones, en los medios especializados en negocios, sobre el débil desempeño del Mercado Alternativo de Valores (MAV), sin ofrecer soluciones que ataquen el problema principal. Por el contrario, la Superintendencia del Mercado de Valores (SMV), ha tomado medidas cortoplacistas, al flexibilizar el reglamento del MAV, que terminarán por atender únicamente los síntomas y cuestionarnos la efectividad de su labor de difusión y promoción del mercado de valores local. En ese contexto, buscaremos contestar qué es lo que realmente hay detrás del débil desempeño del MAV; y veremos que no es responsabilidad única de la SMV, sino que se trata de un desafío mayor - más allá del MAV - pues se tiene que mejorar sustancialmente la gestión profesional de las empresas peruanas, especialmente de las pequeñas y medianas. Con ese objetivo iniciaremos por comprender cómo funciona el mercado de valores, así como los factores que determinan su oferta y demanda para luego centrarnos en el MAV.

Como sabemos, usualmente las empresas recurren al sistema financiero (e.g.; bancos, financieras, cajas municipales), para financiar sus operaciones. En la medida que vayan creciendo, estas empresas necesitarán de mayores montos de dinero para poder ampliar su capacidad productiva, comprar o renovar una nueva línea de producción, entre otros. En ese ciclo de crecimiento, las empresas necesitarán de mucho más dinero, pues ya no son pequeñas empresas; ahora son grandes empresas y podrían conseguir esas grandes necesidades de recursos a menor costo en un mercado financiero mayorista. Este mercado es el mercado de valores, al cual acceden las empresas cuando el sistema financiero no les brinda las mejores soluciones según las necesidades de financiamiento.

SEGÚN LA BOLSA DE VALORES DE LIMA (BVL), EL MAV ES EL SEGMENTO DE LA BOLSA DIRIGIDO A LAS PEQUEÑAS Y MEDIANAS EMPRESAS PERUANAS' QUE FACILITA EL ACCESO A FINANCIAMIENTO EN EL MERCADO BURSÁTIL A MENORES COSTOS Y CON MENORES REQUERIMIENTOS Y OBLIGACIÓN DE INFORMACIÓN.
En este contexto, el mercado de valores se caracteriza, en esencia, por el acceso a importantes cantidades de dinero a tasas de interés competitivas para las empresas que participan del mismo a cambio del cumplimiento de ciertos estándares que las obliga a mantener elevados niveles de transparencia. Tradicionalmente, las grandes empresas y/o aquellas que se encuentra en el denominado segmento de banca corporativa son las que acceden al mercado de valores en búsqueda de capital. Sin embargo, la SMV en su búsqueda por democratizar las bondades del mercado de valores y, por ende, que no sea exclusivo de las grandes empresas, creó hace cuatro años el MAV como una fuente adicional de financiamiento para las pequeñas y medianas empresas que usualmente recurren al sistema financiero en búsqueda de recursos para fondear sus actividades. Según la Bolsa de Valores de Lima (BVL), el MAV es el segmento de la bolsa dirigido a las pequeñas y medianas empresas peruanas ${ }^{1}$ que facilita el acceso a financiamiento en el mercado bursátil a menores costos y con menores requerimientos y obligación de información. En otras palabras, la SMV creó un régimen especializado para las pequeñas y medianas empresas en base a menores requisitos y costos a diferencia del régimen general.

Cabe precisar que los recursos que obtienen las empresas provienen de diferentes inversionistas, entre los que se encuentran fondos de pensiones, compañías de seguros, fondos mutuos, fondos de inversiones, personas naturales con alto patrimonio (i.e., inversionistas retail), entre otros, que depositan su confianza al invertir en dichas empresas para lo cual requieren de una adecuada y fluida información, tanto cuantitativa como cualitativa, con el objetivo de gestionar adecuadamente sus portafolios. Es por ello que la regulación de la SMV solicita que las empresas cumplan con estándares mínimos de transparencia a su vez que los inversionistas demandan el ejercicio de las mejores prácticas de gobierno corporativo. A cambio, las empresas cuentan con una alternativa de financiamiento que, a diferencia del sistema bancario tradicional, le ofrece tasas de interés competitivas, mayores plazos y montos, así como una estructura de financiamiento a la medida (bullet, amortizaciones parciales, tasa de interés variables y dependiendo del emisor, menores garantías, entre otros). Asimismo, producto del cumplimiento de estándares de gestión más exigentes se incrementa el valor, imagen y la visibilidad de la empresa. 
En el régimen general, las empresas necesitan grandes cantidades de dinero para concretar sus planes de crecimiento y cuentan con la estructura necesaria para poder acceder al mercado de valores al contar con estados financieros auditados por firmas internacionales; un área dedicada para el contacto con los inversionistas; clasificaciones de riesgo superiores a AA- en la escala local; una plana gerencial profesional y con experiencia; así como el cumplimiento de mínimas prácticas de gobierno corporativo, entre muchas otras. También, es natural que estas empresas participen del mercado de valores internacional como lo han realizado durante los últimos años ante la coyuntura pasada de bajas tasas de interés. Estas grandes empresas son las que presentan menores problemas ante una primera incursión en el mercado de valores, producto de las relaciones con diferentes acreedores financieros que exigen similares y/o iguales estándares al del mercado de valores. Así, el incursionar por primera vez en el mercado de valores significa una transformación significativa de la empresa que acarrea unos costos de implementación como los de estructuración y adecuación interna a la normativa de la $\mathrm{SMV}$, pero incrementa el valor de la empresa sobremanera.

\section{..QUE PARTICIPAR EN EL MERCADO DE VALORES ES UNA DECISIÓN ESTRATÉGICA MUY IMPORTANTE PARA LO CUAL EL COMPROMISO DE LA ALTA DIRECCIÓN ES CRÍTICO.}

Es por ello que participar en el mercado de valores es una decisión estratégica muy importante para lo cual el compromiso de la alta dirección es crítico. El acceso al mercado de valores implica un hito importante en la historia de la empresa, pues la empresa se hará pública. Tendrá que publicar cada trimestre sus estados financieros; una vez al año una memoria y estados financieros auditados; y cualquier hecho de importancia que pueda afectar el desempeño de la empresa. Asimismo, estará bajo las preguntas y el análisis de los diferentes inversionistas, mientras trabaja con los estructuradores y abogados, así como con las clasificadoras de riesgo. En otras palabras, la empresa pasa a ser objeto de escrutinio por los diferentes agentes del mercado, motivo por el cual algunos empresarios prefieren mantener a la empresa en el ámbito privado y así no tener que brindar información a la competencia. En otros casos, no se hace necesaria la incursión en el mercado de valores local, a pesar de contar con el tamaño y la estructura necesaria, pues su casa matriz puede acceder al mismo capital en mejores condiciones. En cualquiera de los casos, participar o no del mercado de valores, es una decisión ejecutiva de la alta dirección.
Por su parte, los inversionistas buscan obtener los mayores rendimientos posibles según los riesgos que están dispuestos a asumir en función de sus políticas y estrategias de inversión. Para ello, analizan cada nueva alternativa que se les presenta. Cuantitativamente, evalúan la capacidad de generación de flujo de caja de la empresa, los márgenes de rentabilidad, niveles de endeudamiento y liquidez; el tamaño de operaciones, la diversificación de ingresos, entre otros indicadores financieros que luego serán comparados con sus pares directos a fin de establecer el rendimiento a exigir. A la evaluación cuantitativa se suma el análisis cualitativo que considera el respaldo patrimonial que pueda tener la empresa bajo análisis; el sector en el cual realiza sus operaciones; la experiencia y profesionalismo de su plana gerencial; los niveles de gobierno corporativo; $\mathrm{y}$ el liderazgo de los productos o servicios que ofrece, así como el carácter de la alta dirección y otros factores que dependerán de cada evaluación que se realice. En esta etapa inicial de admisión y en la posterior de monitoreo, por agrupar en dos fases el proceso de inversión, es crítica e indispensable la información, financiera o no, para poder realizar el análisis de la oportunidad de inversión, así como tomar las acciones pertinentes con el objetivo de que no se vean afectados los portafolios que administran los inversionistas ante las diferentes variables que pueden afectar el desempeño de las empresas en las que se encuentran invertidos.

Es en ese sentido que la SMV cobra importancia al regular y supervisar la transparencia en la información dentro del mercado de valores para que los inversionistas puedan gestionar sus portafolios de la mejor manera posible (diligente). Adicionalmente y no menos importante, es el rol fiduciario que caracteriza a los inversionistas locales, pues gestionan dinero de terceros lo cual configura uno de los principales motivos por los que son regulados. Es decir, los principales inversionistas del mercado local (e.g., fondos de pensiones, compañías de seguros, fondos mutuos), no invierten su dinero; por el contrario, arriesgan y exigen una rentabilidad por el dinero de sus clientes a cambio de una comisión por su gestión. Este rol fiduciario que tienen los inversionistas está regulado y supervisado por lo cual cada uno de ellos realiza las diligencias del caso

ES EN ESE SENTIOO QUE LA SMV COBRA IMPORTANCIA AL REGULAR Y SUPERVISAR LA TRANSPARENCIA EN LA INFORMACIÓN DENTRO DEL MERCADO DE VALORES PARA QUE LOS INVERSIONISTAS PUEDAN GESTIONAR SUS PORTAFOLIOS DE LA MEJOR MANERA POSIBLE... 
por trabajar con dinero ajeno. Así, la labor que realizan los principales inversionistas es sofisticada y de importante valor para los dueños de los fondos que ellos gestionan por lo cual exigen el mejor de los estándares a las empresas en las cuales invierten.

En ese contexto, donde existe un mercado de valores peruano caracterizado por tener inversionistas sofisticados y acostumbrados a invertir en empresas grandes con determinado nivel de riesgo, la SMV creó el MAV. Sin embargo y como era de esperarse, los principales inversionistas no han participado directamente de las emisiones del MAV en la medida en que el nivel de riesgo, el tamaño de las emisiones, los bajos niveles de gobierno corporativo y el poco track record de dichas empresas en el mercado de valores no logran encajar con los estándares de sus inversiones. Naturalmente, las empresas del MAV y aquellas potenciales empresas para este segmento son de menor tamaño que las que tradicionalmente están acostumbrados los principales inversionistas. El menor tamaño de dichas empresas conllevan riesgos que son parte del ciclo de vida de toda empresa, pues usualmente se encuentran concentradas tanto por cliente como por proveedor; la gestión es liderada por la familia accionista con las características que conocemos de las empresas familiares; la información financiera no contiene el detalle al cual están acostumbrados los inversionistas; algunas empresas no cuentan con directorios y en caso que los tengan, no todas cuentan con directores independientes, entre otros.

Bajo el escenario de que alguna de las empresas del MAV supere los principales puntos de mejora detallados, los montos que necesitan no son suficientes como para que amerite, por lo menos un análisis preliminar por parte de los principales inversionistas. Los montos de las emisiones en el MAV, en línea con el tamaño de las empresas, son pequeños y en caso los inversionistas decidan invertir, atraídos únicamente por la rentabilidad, el retorno asociado a dicha inversión sería marginal y no aumentaría o contribuiría al retorno de todo el portafolio en agregado. Por el contrario, al ser pequeño el peso de la nueva inversión dentro del portafolio, esta se diluiría, con lo cual se habría hecho un uso ineficiente del tiempo y de los recursos del equipo que participa en el proceso de inversiones, toda vez que se puede evaluar una empresa de mayor tamaño en el mismo tiempo. Los principales inversionistas administran grandes cantidades de dinero que con tranquilidad podrían comprar cualquier empresa que participa del MAV únicamente usando los saldos de caja de sus portafolios. ${ }^{2}$

Sin embargo, se está desarrollando el segmento de inversionistas retail que se alinea con la oferta de instrumentos financieros del MAV pues ellos invierten y asumen el riesgo por su propia cuenta a diferencia de los principales inversionistas que poseen un rol fiduciario.
Asimismo, existen algunos fondos de inversión destinados a comprar los valores que se emitan dentro del MAV. Si bien los principales inversionistas no consideran atractivo el MAV, los inversionistas con menor tamaño en sus portafolios observan con interés este nuevo segmento. Con ello queda descartado que el débil desempeño del MAV se deba exclusivamente por factores de demanda, pues existe dicha demanda. No en los niveles que corresponde a una demanda de los principales inversionistas, pero sí acorde a las necesidades de las pequeñas y medianas empresas.

A nivel de oferta, es decir, empresas que ingresaban al MAV, sólo tuvo una respuesta de doce empresas durante los cuatro años de vigencia del MAV. Si bien se considera que este segmento es reciente, los diferentes participantes del mercado de valores consideran bajo este número al tener en cuenta el total de potenciales empresas no supervisadas por la SMV que podrían acceder a este segmento. El poco interés por participar del mercado de valores, según BCRP (2013), a raíz del lanzamiento del MAV, se explica en dos variables: i) los elevados costos de entrada; y ii) la falta de información sobre el mercado de valores. Sobre la primera valla, ésta se ha bajado con los menores costos asociados a una emisión en el MAV, en comparación en el régimen general. En relación a la falta de información, es de esperar que con la promoción del MAV, tanto por la BVL, como la SMV, el desconocimiento sobre el mismo haya disminuido entre los potenciales emisores $^{3}$. Entonces, surge la interrogante de qué es lo que realmente frena a los principales directivos a participar del mercado de valores a través del MAV, dado que las barreras se han bajado y ahora tienen mayor conocimiento de las bondades del mercado de capitales.

\section{SI REALMENTE QUEREMOS SOLUCIONAR EL PROBLEMA PRINCIPAL SE TIENEN QUE TOMAR MEDIDAS CUYOS RESULTADOS SE OBSERVEN EN EL LARGO PLAZO.}

Ensayando una respuesta, ella se debería a que los directivos de dichas empresas no desean participar, ya sea porque no cuentan con la infraestructura necesaria para cumplir con los estándares que se requieren, o ante el poco conocimiento del mismo, tienen temor de hacer pública su información financiera. En cualquiera de los dos escenarios, los principales directivos de dichas empresas no están decidiendo a favor de participar en el mercado de valores al igual que sucede en las grandes empresas, pero por diferentes motivos. También, es de esperar que los directivos no vean incentivos tangibles de incursionar en el MAV, pues los requerimientos de 
capital los puede otorgar el sistema bancario tradicional e inicialmente se pueden pagar tasas superiores a las que pagan actualmente. En ese sentido y dado que la balanza se inclina más por un problema de oferta para explicar el débil desempeño del MAV ¿qué se puede hacer para revertir la situación actual?

Se pueden tomar diferentes y relativamente rápidas medidas que ayudarían al dinamismo del MAV, como la reciente modificación de la norma ${ }^{4}$, pero éstas atacarían síntomas de corto plazo. Si realmente queremos solucionar el problema principal se tienen que tomar medidas cuyos resultados se observen en el largo plazo. El hecho de que las pequeñas y medianas empresas no sean consideradas por los principales inversionistas y que sean pocas las empresas que participan del MAV, tienen como principal desafío el educar y profesionalizar la gestión financiera de las pequeñas y medianas empresas pues tienen gran relevancia para la economía peruana, toda vez que el financiamiento del sistema financiero a dichas empresas representa el 17.2\% del PBI (SBS, 2015). Asimismo, se debe buscar mejorar los niveles de gobierno corporativo y transparencia en la información para que todos los inversionistas, con deber fiduciario o no, puedan invertir en dichas empresas. Es un trabajo de largo plazo pero es necesario en la medida en que beneficiará a todos.

1. Según la resolución $\mathrm{N}^{\circ}$ 006-2016-SMV/01, con una facturación inferior a los S/.350.0 millones.

2. Al cierre del 2015, únicamente las AFP administraban S/.123 mil millones

3. No se cuenta con un estudio público definitivo sobre el conocimiento del mercado de valores por parte de los directivos a cargo de las empresas objetivo del MAV.

4. Se subió el umbral de S/.200.0 a S/.350.0 millones al monto máximo de facturación que la empresa debe cumplir para ser incluida en el MAV, así como la eliminación de la clasificación de riesgo para instrumentos de corto plazo que se emitan siempre y cuando el emisor se encuentre calificado como normal ante la SBS.

\section{REFERENCIAS}

BCRP. (9 de Abril de 2013). Notas de Estudio del BCRP(21). Lima, Perú.

SBS. (Diciembre de 2015). Carpeta de Cuadros Estadísticos del Sistema Financiero. Obtenido de Superintendencia de Banca, Valores y Seguros: http://www.sbs.gob.pe/app/ stats/EstadisticaBoletinEstadistico.asp?p=14\# 